\title{
OPTIMAL PRICING OF A HETEROGENEOUS PORTFOLIO FOR A GIVEN RISK LEVEL
}

\author{
BY \\ Yaniv Zaks, Esther Frostig And Benny LeviKson ${ }^{1}$
}

\begin{abstract}
Consider a portfolio containing heterogeneous risks, where the policyholders' premiums to the insurance company might not cover the claim payments. This risk has to be taken into consideration in the premium pricing. On the other hand, the premium that the insureds pay has to be fair. This fairness is measured by the distance between the risk and the premium paid. We apply a non-linear programming formulation to find the optimal premium for each class so that the risk is below a given level and the weighted distance between the risk and the premium is minimized. We consider also the dual problem: minimizing the risk level for a given weighted distance between risks and premium.
\end{abstract}

\section{KEYWORDS}

Heterogeneous portfolio, Positive Definite Matrix, non-linear programming, dual problem.

\section{INTRODUCTION}

Consider a portfolio containing independent identically distributed (i.i.d) risks. When pricing the insurance policy for such risks, the following two principles are usually taken into consideration:

1. The probability that the total claims exceed the total premiums paid is a predetermined small number, $\alpha, 0<\alpha<1$.

2. The premium increases as the expected claim size increases.

In this paper we consider a portfolio with $k$ classes of insureds, each containing a large and fixed number of insureds.

1 This article is dedicated to the memory of our beloved friend Professor Benjamin Zeev Levikson who passed away on July, 16, 2005. 
The goal is to find a premium for each class such that principle 1 will hold and such that the premiums vector $\pi=\left(\pi_{1}, \cdots, \pi_{k}\right)$ will minimize the expected squared distance between the total claim amount and the total premiums income in each class. Here, $\pi_{j}$ is the premium for class $j$. It will be shown that under this optimization scheme principle 2 also holds, within each class.

Bowers et al. (Chapter, 2.5, 1997) suggest the following premium for each class $j: \pi_{j}=\left(1+\theta_{\alpha}\right) \mu_{j}$, where $\mu_{j}$ is the expected risk of class $j$, and $\theta_{\alpha}$ is a loading factor which is determined so that the probability that the total claims exceed the total premiums paid is $\alpha$. This method is called the expected principle.

Some other premium pricing methods determine the premium only according to principle 2, which is proportional to the expected claim size, without considering principle 1. Lemaire (1995) demonstrates a Bonus-Malus System (BMS) that is based only on the number of the claims. Calculations of the BMS are based on the Bayes' rule. Frangos and Vrontos (2001) develop a BMS based on the frequency and the severity of the claims. Both components are determined by the Bayes' theorem. Kliger and Levikson (1998) consider a portfolio having i.i.d claims, from an economic point of view. They find the premium such that the profit is maximized taking into account the number of policyholders. Goovaerts et al. (1984) present properties of premium calculation principles.

The innovation in this paper is a systematic method to obtain the premium for each class. By applying this method, the insurer has the flexibility to price the premium for each class according to the company's policy.

In section 2 we define the risk in terms of probability of ruin and then we discuss a homogeneous portfolio consisting of i.i.d. risks. The main results are in sections 3 and 4 . In section 3 we analyze heterogeneous portfolio, where the risks are not identically distributed. In Section 4 we state and solve the dual problem.

\section{Preliminaries}

In this section we define homogeneous and heterogeneous portfolios. We describe intuitive and known methods to price premiums in both cases.

\subsection{A homogeneous portfolio}

Consider a portfolio containing $n$ risks $X_{1}, \cdots, X_{n}$. Let $\pi_{i}$ be the premium price for risk $i$.

Definition 1: Let $0<\alpha<1$. An insurance company is faced with a risk level $\alpha$ if the probability that the total claims exceed the total premiums is $\alpha$. That is:

$$
P\left(\sum_{i=1}^{n} \pi_{i} \leq \sum_{i=1}^{n} X_{i}\right)=\alpha .
$$


A portfolio containing $n$ i.i.d risks $X_{i}, i=1, \cdots, n$ with mean $\mu_{X}$ and variance $\sigma_{X}^{2}$ is called a homogeneous portfolio. Clearly, in a homogeneous portfolio all the insurds pay the same premium $\pi$.

Let

$$
S=\sum_{i=1}^{n} X_{i}, \mu_{s}=E[S]=n \mu_{X}, \text { and } \sigma_{S}^{2}=\operatorname{Var}(S)=n \sigma_{X}^{2} .
$$

Assume that the company is ready to face a risk $\alpha$, that is

$$
P(S \geq n \pi) \leq \alpha .
$$

By the Central Limit Theorem, for a sufficient large $n$

$$
\frac{S-\mu_{S}}{\sigma_{S}} \sim N(0,1)
$$

Thus the minimal premium is (See Bowers et. al. (1997), Chapter 2.5)

$$
\pi=\mu_{X}+z_{1-\alpha} \frac{\sigma_{X}}{\sqrt{n}},
$$

where $z_{1-\alpha}$ is the $1-\alpha$ percentile of the standard normal distribution.

\subsection{A heterogeneous portfolio}

Throughout the paper we consider a portfolio composed of $k$ risk classes. We call this portfolio a heterogeneous portfolio. The following assumptions hold:

(a) The risks in the portfolio are independent.

(b) Class $j$ contains $n_{j}$ i.i.d risks, $X_{j, 1} \cdots, X_{j, n_{j}}$ distributed as $X_{j}$, with mean $\mu_{j}$ and variance $\sigma_{j}^{2}, j=1, \cdots, k$. Let $n=\sum_{j=1}^{k} n_{j}$.

(c) Assume that $n_{j}, j=1, \cdots, k$, are large enough to implement the central limit theorem (CLT).

For any random variable $X$ let $X^{*}=\frac{X-E[X]}{\sqrt{\operatorname{var}[X]}}$.

The total risk of class $j, S_{j}$ is $S_{j}=\sum_{i=1}^{n_{j}} X_{j, i}$. Clearly, $E\left[S_{j}\right]=n_{j} \mu_{j}$ and $\operatorname{Var}\left[S_{j}\right]=n_{j} \sigma_{j}^{2}$.
By CLT $S_{j}^{*} \sim N(0,1)$.

Let $S=\sum_{j=1}^{k} S_{j}$, with mean $\mu$ and variance $\sigma^{2}$, where

$$
\mu=E[S]=\sum_{i=1}^{k} n_{i} \mu_{i} \text { and } \sigma^{2}=\operatorname{var}[S]=\sum_{i=1}^{k} n_{i} \sigma_{i}^{2} .
$$


Furthermore, by CLT. $S^{*} \sim N(0,1)$.

Next, we describe some approaches to determine the premium for each class.

\subsubsection{The individual approach}

The insurer specifies for each class $j$, its risk level $\alpha_{j}$. For each class the premium is calculated as in equation (2), i.e.,

$$
\pi_{j}=\mu_{j}+z_{1-\alpha_{j}} \frac{\sigma_{j}}{\sqrt{n_{j}}}
$$

This approach considers only the size of each class $j, j=1, \cdots, k$ and does not consider the effect of the total size of the population.

\subsubsection{The global approach}

The insurer determines the premium for each class such that the risk level is $\alpha$, i.e., principle 1 holds, and principle 2 holds (for each class). Thus, similar to (1),

$$
P\left(\sum_{j=1}^{k} n_{j} \pi_{j} \leq \sum_{j=1}^{k} S_{j}\right)=\alpha
$$

In view of assumption (c) we obtain:

$$
\sum_{j=1}^{k} n_{j} \pi_{j}=\mu+z_{1-\alpha} \sigma=\sum_{j=1}^{k} n_{j} \mu_{j}+z_{1-\alpha} \sqrt{\sum_{j=1}^{k} n_{j} \sigma_{j}^{2}} .
$$

Equation (3) presents the total amount of premiums which the insurer should collect in order to achieve a risk level $\alpha$.

Remark: Let us compare the total premium collected according to the individual approach, and to the global approach.

The inequality, $\sum a_{i}^{2} \leq\left(\sum a_{i}\right)^{2}$ where $a_{i}>0$ implies that

$$
\begin{aligned}
& \sum_{i=1}^{k} n_{i} \mu_{i}+z_{1-\alpha} \sqrt{\sum_{i=1}^{k} n_{i} \sigma_{i}^{2}}<\sum_{i=1}^{k} n_{i} \mu_{i}+z_{1-\alpha} \sum_{i=1}^{k} \sigma_{i} \sqrt{n_{i}} \\
& =\sum_{i=1}^{k} n_{i}\left(\mu_{i}+z_{1-\alpha} \frac{\sigma_{i}}{\sqrt{n_{i}}}\right)
\end{aligned}
$$

The left hand side of (4) is the sum of all premiums which are collected according to the global approach for a risk level $\alpha$. The last term of (4) is the sum 
of all premiums which are collected according to the individual approach by taking the same risk level $\alpha$ for each class. Thus, in the global approach, the policyholders pay less than in the individual approach.

It still remains to set the premium for each class. One tends to price the premium of class $i$ as follows:

$$
\pi_{i}=\mu_{i}+z_{1-\alpha} T_{i}
$$

where $T_{1}, \ldots, T_{k}$ are nonnegative numbers such that

$$
\sum_{i=1}^{k} n_{i} T_{i}=\sqrt{\sum_{i=1}^{k} n_{i} \sigma_{i}^{2}}=\sigma
$$

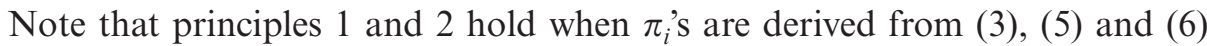
for $i=1, \cdots, k$.

Clearly, there is an infinite number of options to determine $T_{1}, \ldots, T_{k}$. In what follows we present some of them.

\section{(1) Uniform allocation}

The global standard deviation $\sigma$ defined in (6), is split equally among all the policyholders, that is $T_{1}=\ldots=T_{k}=\frac{\sigma}{n}$. The premium for a policyholder in class $j$ is

$$
\pi_{j}=\mu_{j}+z_{1-\alpha} \frac{\sigma}{n} .
$$

\section{(2) Semi-Uniform allocation}

The global standard deviation $\sigma$ is split equally among the classes. Here $T_{i}=\frac{\sigma}{k n_{i}}$. The premium for a policyholder in class $j$ is

$$
\pi_{j}=\mu_{j}+z_{1-\alpha} \frac{\sigma}{k n_{j}} .
$$

\section{(3) Proportional allocation}

The global variance $\sigma^{2}=\sum_{j=1}^{k} n_{j} \sigma_{j}^{2}$ is split among the classes proportionally to the variance of the classes. Thus, $T_{i}$ is determined as $\frac{r_{i} \sigma}{n_{i}}$, where $r_{i}=\frac{n_{i} \sigma_{i}^{2}}{\sigma^{2}}$. The premium for a policyholder in class $i$ is

$$
\pi_{i}=\mu_{i}+z_{1-\alpha} \frac{r_{i} \sigma}{n_{i}} .
$$

The next section contains the main result of the paper. We describe a systematic approach to price class $j$ premium such that principle 1, i.e. (3), and principle 2 hold. All the pricing schemes described above will be derived by this general approach. 


\section{The optimal ALLOCATiON}

In this section we determine the premium for each class such that the following two conditions hold:

1. Principle 1 holds. Note that under our assumptions principle 1 is equivalent to the constraint given by (3).

2. The sum of the expected weighted squared distances between each class risk, $S_{j}$, and class premium, $n_{j} \pi_{j}$, i.e. $\sum_{j=1}^{k} \frac{1}{r_{i}} E\left(S_{j}-n_{j} \pi_{j}\right)^{2}$, is minimized.

\subsection{The analysis and the solution of the non-linear optimization problem}

\section{Theorem 1}

Let $\pi=\left(\pi_{1}, \cdots, \pi_{k}\right)$, and $r_{1}, \ldots, r_{k}$ be positive numbers. The minimization problem

$$
\text { (I) }\left\{\begin{array}{l}
\min _{\pi}\left\{\sum_{i=1}^{k}\left[\frac{1}{r_{i}} E\left(S_{i}-n_{i} \pi_{i}\right)^{2}\right]\right\} \\
\text { s.t. } \sum_{i=1}^{k} n_{i} \pi_{i}=\mu+z_{1-\alpha} \sigma
\end{array}\right.
$$

has a unique solution which is

$$
\pi_{j}=\mu_{j}+z_{1-\alpha} \frac{r_{j} \sigma}{r n_{j}},
$$

where $r=\sum_{j=1}^{k} r_{j}$.

To prove Theorem 1 we need two lemmas on positive definite matrices. The proofs of these two lemmas appear in the Appendix.

\section{Lemma 1}

Let $A$ be a positive definite matrix and let $P$ be a nonsingular matrix, both of dimensions $m \times m$. Let $P^{t}$ be the transpose matrix of $P$. Then $B=P^{t} A P$ is a positive definite matrix.

\section{Lemma 2}

Let $a_{1}, \ldots, a_{m}$ be positive numbers, and let the matrix $A_{m}$ be as follows:

$$
A_{m}=\left[\begin{array}{cccc}
1+a_{1} & 1 & \cdots & 1 \\
1 & 1+a_{2} & \ddots & \vdots \\
\vdots & \ddots & \ddots & 1 \\
1 & \cdots & 1 & 1+a_{m}
\end{array}\right]
$$

Then the matrix $A_{m}$ is a positive definite matrix. 


\section{Proof of Theorem 1}

Note that for $i=1, \cdots, k$

$$
\begin{aligned}
n_{i} \sigma_{i}^{2} & =\operatorname{Var}\left(S_{i}\right)=\operatorname{Var}\left(S_{i}-n_{i} \pi_{i}\right) \\
& =E\left(S_{i}-n_{i} \pi_{i}\right)^{2}-E^{2}\left(S_{i}-n_{i} \pi_{i}\right) \\
& =E\left(S_{i}-n_{i} \pi_{i}\right)^{2}-\left(n_{i} \mu_{i}-n_{i} \pi_{i}\right)^{2} .
\end{aligned}
$$

Hence

$$
E\left(S_{i}-n_{i} \pi_{i}\right)^{2}=n_{i} \sigma_{i}^{2}+\left(n_{i} \mu_{i}-n_{i} \pi_{i}\right)^{2} .
$$

Substituting (7) in the objective function in problem (I), yields the following:

$$
\begin{aligned}
& \min _{\pi}\left\{\sum_{i=1}^{k}\left[\frac{1}{r_{i}} E\left(S_{i}-n_{i} \pi_{i}\right)^{2}\right]\right\}= \\
= & \min _{\pi}\left\{\sum_{i=1}^{k}\left[\frac{1}{r_{i}}\left(n_{i} \sigma_{i}^{2}+\left(n_{i} \mu_{i}-n_{i} \pi_{i}\right)^{2}\right)\right]\right\} \\
= & \min _{\pi}\left\{\sum_{i=1}^{k} \frac{n_{i} \sigma_{i}^{2}}{r_{i}}+\sum_{i=1}^{k} \frac{1}{r_{i}}\left(n_{i} \mu_{i}-n_{i} \pi_{i}\right)^{2}\right\} .
\end{aligned}
$$

Clearly, eliminating the expression $\sum_{i=1}^{k} \frac{n_{i} \sigma_{i}^{2}}{r_{i}}$ does not change the optimal solution.

Let $W_{i}(\pi)=n_{i} \mu_{i}-n_{i} \pi_{i}, 1 \leq i \leq k$. Problem (I) is equivalent to the following problem (II):

$$
\text { (II) }\left\{\begin{array}{l}
\min _{\pi}\left\{\sum_{i=1}^{k}\left[\frac{1}{r_{i}} W_{i}^{2}(\pi)\right]\right\} \\
\text { s.t. } \sum_{i=1}^{k} W_{i}(\pi)=-z_{1-\alpha} \sigma
\end{array}\right. \text {. }
$$

The constraint in problem (II) can be rewritten as follows:

$$
-W_{1}(\pi)=\sum_{i=2}^{k} W_{1}(\pi)+z_{1-\alpha} \sigma .
$$

Let $G(\pi)=\sum_{i=1}^{k} \frac{1}{r_{i}} W_{i}^{2}(\pi)$. Substituting $(8)$ in $G(\pi)$ yields:

$$
G(\pi)=\frac{1}{r_{1}}\left(\sum_{i=2}^{k} W_{1}(\pi)+z_{1-\alpha} \sigma\right)^{2}+\sum_{i=2}^{k} \frac{1}{r_{i}} W_{i}^{2}(\pi) .
$$


Thus, problem (II) is equivalent to problem (III), where

$$
\text { (III) } \min _{\pi}\{G(\pi)\} \text {. }
$$

First we find the extreme points of $G(\pi)$, that is, the vectors $\pi$ for which all the first partial derivatives vanish.

Note that $G(\pi)$ is a function of $\pi_{2}, \ldots, \pi_{k}$. The first partial derivatives are:

$$
\frac{\partial G}{\partial \pi_{i}}=\frac{-2 n_{i}}{r_{1}}\left(\sum_{i=2}^{k} W_{1}(\pi)+z_{1-\alpha} \sigma\right)-\frac{2 n_{i}}{r_{i}} W_{1}(\pi) \quad \forall i=2, \ldots, k
$$

Equating the k-1 partial derivatives in (10) to 0 yields the following system of linear equations for $W_{2}(\pi), \ldots, w_{k}(\pi)$ :

$$
\left\{\begin{array}{l}
\left(\frac{1}{r_{1}}+\frac{1}{r_{2}}\right) W_{2}+\frac{1}{r_{1}} \sum_{\substack{i=2 \\
i \neq 2}}^{k} W_{i}=-\frac{z_{1-\alpha} \sigma}{r_{1}} \\
\left(\frac{1}{r_{1}}+\frac{1}{r_{3}}\right) W_{3}+\frac{1}{r_{1}} \sum_{\substack{i=2 \\
i \neq 3}}^{k} W_{i}=-\frac{z_{1-\alpha} \sigma}{r_{1}} \\
\vdots \\
\left(\frac{1}{r_{1}}+\frac{1}{r_{k}}\right) W_{k}+\frac{1}{r_{1}} \sum_{\substack{i=2 \\
i \neq k}}^{k} W_{i}=-\frac{z_{1-\alpha} \sigma}{r_{1}}
\end{array},\right.
$$

where $W_{i}=W_{i}(\pi)$. Subtracting the first equations from each of the other equations yields the following set of linear equations (written in matrix form)

$$
\left[\begin{array}{cccccc:c}
\frac{1}{r_{1}}+\frac{1}{r_{2}} & \frac{1}{r_{1}} & \cdots & \cdots & \cdots & \frac{1}{r_{1}} & -\frac{z_{1-\alpha} \sigma}{r_{1}} \\
\frac{1}{r_{2}} & -\frac{1}{r_{3}} & 0 & \cdots & \cdots & 0 & 0 \\
\vdots & 0 & \ddots & & 0 & \vdots & \vdots \\
\vdots & \vdots & \ddots & \ddots & & \vdots & \vdots \\
\vdots & \vdots & 0 & \ddots & \ddots & 0 & \vdots \\
\frac{1}{r_{2}} & 0 & \cdots & \cdots & 0 & -\frac{1}{r_{k}} & 0
\end{array}\right] .
$$

The last $\mathrm{k}-2$ rows yield that

$$
r_{2} W_{i}=r_{i} W_{2} \quad \forall i=3, \ldots, k .
$$

The first row presents the equation 


$$
\left(\frac{1}{r_{1}}+\frac{1}{r_{2}}\right) W_{2} \frac{1}{r_{1}} \sum_{i=3}^{k} W_{1}=-\frac{z_{1-\alpha} \sigma}{r_{1}} .
$$

By substituting (11) in (12) and then multiplying (12) by $r_{1} r_{2}$ we obtain

$$
\sum_{i=1}^{k} r_{i} W_{2}=-r_{2} z_{1-\alpha} \sigma .
$$

Hence,

$$
W_{2}=\frac{-r_{2} z_{1-\alpha} \sigma}{r}
$$

Substituting (13) in (11) yields,

$$
W_{i}=\frac{-r_{i} z_{1-\alpha} \sigma}{r}, \quad i=3, \ldots, k
$$

Finally, substituting (13) and (14), in (8) yields

$$
W_{1}=\frac{r_{1} z_{1-\alpha} \sigma}{r} .
$$

Since $W_{i}(\pi)=n_{i} \mu_{i}-n_{i} \pi_{i}$ the extreme point $\pi^{*}=\left(\pi_{1}^{*}, \ldots, \pi_{k}^{*}\right)$ for problem (III) is

$$
\pi_{i}^{*}=\mu_{i}+z_{1-\alpha} \frac{r_{i} \sigma}{r n_{i}}, \quad i=1, \ldots, k
$$

Next we have to verify that the Hessian matrix of the function given by (9), at $\pi^{*}$ is a positive definite matrix. By differentiating (10), we find the second partial derivatives of $G(\pi)$ :

$$
\begin{gathered}
\frac{\partial^{2} G}{\partial \pi_{i}^{2}}=\frac{2 n_{i}^{2}}{r_{1}}+\frac{2 n_{i}^{2}}{r_{i}}=\frac{2 n_{i}^{2}\left(r_{1}+r_{i}\right)}{r_{1} r_{i}}, \quad i=2, \ldots, k \\
\frac{\partial^{2} G}{\partial \pi_{j} \partial \pi_{i}}=\frac{2 n_{i} n_{j}}{r_{1}} \quad 2 \leq i<j \leq k .
\end{gathered}
$$

Therefore the Hessian matrix, at all points, is

$$
H=\left[\begin{array}{cccccc}
\frac{2 n_{2}^{2}\left(r_{1}+r_{2}\right)}{r_{1} r_{2}} & \frac{2 n_{2} n_{3}}{r_{1}} & \frac{2 n_{2} n_{4}}{r_{1}} & \ldots & \ldots & \frac{2 n_{2} n_{k}}{r_{1}} \\
\frac{2 n_{3} n_{2}}{r_{1}} & \frac{2 n_{3}^{2}\left(r_{1}+r_{3}\right)}{r_{1} r_{3}} & \frac{2 n_{3} n_{4}}{r_{1}} & \ldots & \ldots & \frac{2 n_{3} n_{k}}{r_{1}} \\
\vdots & & \ddots & \ddots & & \\
\vdots & & & & \ddots & \frac{2 n_{k-1} n_{k}}{r_{1}} \\
\vdots & & & & 2 n_{k} n_{k-1} & \frac{2 n_{k}^{2}\left(r_{1}+r_{k}\right)}{r_{1} r_{k}}
\end{array}\right] .
$$


The property of a positive definite is not changed by multiplying the matrix by a positive scalar. Thus, it is sufficient to show that the following matrix is a positive definite:

$$
\tilde{H}=\left[\begin{array}{cccccc}
\frac{n_{2}^{2}\left(r_{1}+r_{2}\right)}{r_{2}} & n_{2} n_{3} & n_{2} n_{4} & \cdots & \cdots & n_{2} n_{k} \\
n_{3} n_{2} & \frac{n_{3}^{2}\left(r_{1}+r_{3}\right)}{r_{3}} & n_{3} n_{4} & \cdots & \cdots & n_{3} n_{k} \\
\vdots & & \ddots & \ddots & & \\
\vdots & & & \ddots & \ddots & n_{k-1} n_{k} \\
n_{k} n_{2} & n_{k} n_{3} & \cdots & \cdots & n_{k} n_{k-1} & \frac{n_{k}^{2}\left(r_{1}+r_{k}\right)}{r_{k}}
\end{array}\right] .
$$

$\tilde{H}$ is a symmetric matrix which fulfills the equality

$$
\tilde{H}=\left[\begin{array}{ccc}
n_{2} & \cdots & 0 \\
\vdots & \ddots & \vdots \\
0 & \cdots & n_{k}
\end{array}\right]\left[\begin{array}{ccc}
1+\frac{r_{1}}{r_{2}} & \cdots & 1 \\
\vdots & \ddots & \vdots \\
1 & \cdots & 1+\frac{r_{1}}{r_{k}}
\end{array}\right]\left[\begin{array}{ccc}
n_{2} & \cdots & 0 \\
\vdots & \ddots & \vdots \\
0 & \cdots & n_{k}
\end{array}\right] .
$$

By Lemma 1 and 2, $\tilde{H}$ is a positive definite matrix and so is the Hessian matrix $H$. Hence $\pi^{*}$ is the unique solution for problem (I).

In Theorem 1 the constraint is equality. In practice the insurer constraint is

$$
P\left(\sum_{j=1}^{k} S_{j} \geq \sum_{j=1}^{k} n_{j} \pi_{j}\right) \leq \alpha, \text { or equivalently } \sum_{i=1}^{k} n_{i} \pi_{i} \geq \mu+z_{1-\alpha} \sigma .
$$

\section{Corolary 1}

Let $\pi=\left(\pi_{1}, \cdots, \pi_{k}\right)$, and let $r_{1}, \ldots, r_{k}$ be positive numbers, The minimization problem

$$
\text { (VI) }\left\{\begin{array}{l}
\min _{\pi}\left\{\sum_{i=1}^{k}\left[\frac{1}{r_{i}} E\left(S_{i}-n_{i} \pi_{i}\right)^{2}\right]\right\} \\
\text { s.t. } \sum_{i=1}^{k} n_{i} \pi_{i} \geq \mu+z_{1-\alpha} \sigma
\end{array}\right.
$$

has $\boldsymbol{a}$ unique solution which is 


$$
\pi_{j}=\mu_{j}+z_{1-\alpha} \frac{r_{j} \sigma}{r n_{j}}, \quad j=1, \cdots, k
$$

where $r=\sum_{j=1}^{k} r_{j}$.

\section{Proof}

Let $\lambda>0$. Consider the following minimization problem:

$$
(\mathrm{V})=\left\{\begin{array}{l}
\min _{\pi}\left\{\sum_{i=1}^{k}\left[\frac{1}{r_{i}} E\left(S_{i}-n_{i} \pi_{i}\right)^{2}\right]\right\} \\
\text { s.t. } \sum_{i=1}^{k} n_{i} \pi_{i}=\mu+\lambda \sigma
\end{array} .\right.
$$

In view of Theorem 1, Problem (V) has the following unique solution: $\pi_{i}=\mu_{i}+$ $\lambda \frac{r_{i} \sigma}{r n_{i}}, i=1, \cdots, k$. Thus substituting $\pi_{i}$ in Equation (7), yields that for a given $\lambda$, the optimal value of the corresponding objective function is:

$$
\sum_{i=1}^{k} \frac{1}{r_{i}}\left(n_{i} \sigma_{i}^{2}+\left(\lambda \frac{r_{i} \sigma}{r}\right)^{2}\right)
$$

The objective function increases in $\lambda$ on $\lambda>0$. Note that the risk level is less or equal to $\alpha$, if, and only if $\lambda \geq z_{1-\alpha}$. Thus the objective function attains its minimum at $\lambda=z_{1-\alpha}$.

Remark: By choosing the appropriate $r_{i}, i=1, \cdots, k$, the insurer has the flexibility to determine the premium for each class according to the company's policy.

\subsection{Applications of Theorem 1}

In this subsection we demonstrate that the various premium pricing methods presented in subsection 2.2.2 can be obtained from Theorem 1 by different choices of $r_{j}, j=1, \cdots, k$.

1. $r_{i}=n_{i}$ for each $i=1, \ldots, k$. It follows that $\sum_{j=1}^{k} r_{j}=\sum_{j=1}^{k} n_{j}=n$. Hence

$$
\pi_{i}^{*}=\mu_{i}+z_{1-\alpha} \frac{r_{i} \sigma}{n_{i} \sum r_{j}}=\mu_{i}+z_{1-\alpha} \frac{\sigma}{n} .
$$

This is the case of the uniform allocation. 
2. $r_{i}=1$ for $i=1, \ldots, k$. In this case $\sum_{j=1}^{k} r_{j}=k$. Hence

$$
\pi_{i}^{*}=\mu_{i}+z_{1-\alpha} \frac{r_{i} \sigma}{n_{i} \sum r_{j}}=\mu_{i}+z_{1-\alpha} \frac{\sigma}{n_{i} k} .
$$

This is the case of the semi-uniform allocation.

3. $r_{i}=\frac{n_{i} \sigma_{i}^{2}}{\sigma^{2}}$ for $i=1, \ldots, k$. Since $\sigma^{2}=\sum_{j=1}^{k} n_{j} \sigma_{j}^{2}$ we obtain $\sum_{j=1}^{k} r_{j}=1$.

Therefore

$$
\pi_{i}^{*}=\mu_{i}+z_{1-\alpha} \frac{r_{i} \sigma_{S}}{n_{i}}
$$

This is the case of the relative allocation.

4. Let $Q$ be a function from the space of all nonnegative random variable to $\mathbf{R}$, such that for independent random variables $X_{1}, \ldots, X_{n}, Q\left(X_{1}+\cdots+X_{k}\right)=\sum_{j=1}^{k} Q\left(X_{j}\right)$, Let $r_{i}=\frac{Q\left(S_{i}\right)}{Q(S)}$. In this case the premium for class $i$, is $\pi_{i}=\mu_{i}+z_{1-\alpha} \frac{\sigma_{S}}{n_{i} Q(S)} Q\left(S_{i}\right)$.

Consider two special cases: 4.1. $\underset{\text { Ciple. }}{Q(X)}=E[X]$. In this case $\pi_{i}=\mu_{i}+z_{1-\alpha} \frac{\sigma}{n_{i} \mu} \mu_{i}$. This is the expectation prin-
.

4.2. $\underset{\text { ciple. }}{Q(X)}=\operatorname{Var}[X]$. In this case $\pi_{i}=\mu_{i}+z_{1-\alpha} \frac{\sigma}{n_{i} \mu} \frac{\sigma_{i}^{2}}{\sigma^{2}}$. This is the variance prin-
.

\subsection{Heterogeneous portfolio with ordered premium}

In this subsection we determine the premium for each class such that principles (1) and (2) hold, and in addition, we consider the case where the insurer or the regulator, wants to grade the premiums for each class such that the premium will increase as its risk increases. We also want to preserve, for each class, the second premium principle as stated in the introduction. Thus, we impose more constraints to the optimization problem (I).

\section{Theorem 2}

Let $a_{1}, \ldots, a_{k-1}$ be nonnegative and $r_{1}, \ldots, r_{k}$ be positive scalars such that

$$
\left(1+a_{i}\right) \mu_{i} \leq \mu_{i+1} \quad i=1, \ldots, k-1 .
$$


The minimization problem

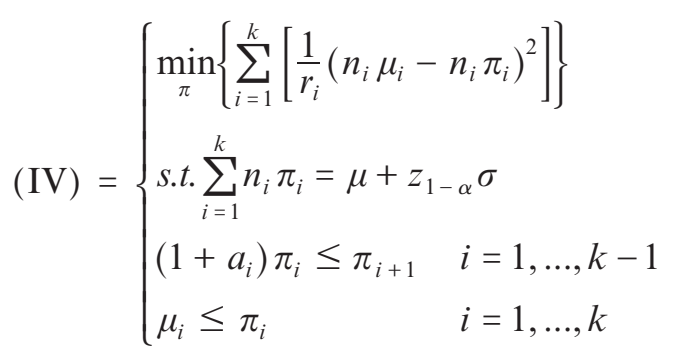

has a unique solution.

Proof

Substituting $Y_{i}=\frac{n_{i} \mu_{i}}{\sqrt{r_{i}}} \quad X_{i}=\frac{n_{i} \pi_{i}}{\sqrt{r_{i}}}$ in problem (IV), yields problem (V) as follows:

$$
(\mathrm{V})= \begin{cases}\min _{\vec{X} \in \mathbb{R}_{+}^{k}}\left\{\sum_{i=1}^{k}\left[\left(Y_{i}-X_{i}\right)^{2}\right]\right\}=\min _{\vec{X} \in \mathbb{R}_{+}^{k}}\left\{\sum_{i=1}^{k}\left[\left(X_{i}-Y_{i}\right)^{2}\right]\right\} \\ \text { s.t. (a) } \sum_{i=1}^{k} \sqrt{r_{i}} X_{i}=\sum_{i=1}^{k} \sqrt{r_{i}} Y_{i}+z_{1-\alpha} \sigma \\ \text { (b) }\left(1+a_{i}\right) \frac{\sqrt{r_{i}} X_{i}}{n_{i}} \leq \frac{\sqrt{r_{i+1}} X_{i+1}}{n_{i+1}} & i=1, \ldots, k-1 \\ \text { (c) } Y_{i} \leq X_{i} & i=1, \ldots, k\end{cases}
$$

Let $W_{i}=X_{i}-Y_{i}$. Hence $(\mathrm{V})$ becomes

$$
(\mathrm{VI})= \begin{cases}\min _{\vec{W} \in \mathbb{R}_{+}^{k}}\left\{\sum_{i=1}^{k} W_{i}^{2}\right\}=\min _{\vec{W} \in \mathbb{R}_{+}^{k}}\|\vec{W}\|^{2} \\ \text { s.t. (a) } \sum_{i=1}^{k} \sqrt{r_{i}} W_{i}=z_{1-\alpha} \sigma \\ \text { (b) }\left(1+a_{i}\right) \frac{\sqrt{r_{i}}\left(W_{i}+Y_{i}\right)}{n_{i}} \leq \frac{\sqrt{r_{i+1}}\left(W_{+1}+Y_{i+1}\right)}{n_{i+1}} & i=1, \ldots, k-1 \\ \text { (c) } 0 \leq W_{i} & i=1, \ldots, k\end{cases}
$$

Constraint (a) is a hyper-plane in $\mathbb{R}^{k}$.

Constraints (b) and (c) are half spaces in $\mathbb{R}^{k}$. 
The intersection of (a), (b) and (c) contains the solution

$$
W_{1}=\ldots=W_{k-1}=0 \quad W_{k}=\frac{z_{1-\alpha} \sigma}{\sqrt{r_{k}}} .
$$

Therefore the constraints define a non-empty convex set. Baptiste et al. (1993, Chapter III.3) study the problem of projection onto a convex set and they prove the existence and uniqueness of such a projection. Consequently, there is a unique solution for problem (VI).

Remark: The objective function of Problem (IV) is to find a point $\vec{W} \in \mathbb{R}_{+}^{k}$ in the region described by the constraints (a)-(c) with minimal Euclidian distance to $\overrightarrow{0}$. The solution can be found using methods of nonlinear programming.

Another method is using Dykstra algorithm (Dykstra, (1983), Censor and Reich, (1998)). Let $C_{j} \subset \mathbb{R}^{n}, j=1, \cdots, m$ be closed and convex sets in $\mathbb{R}^{n}$, and let $C=\bigcap_{j=1}^{m} C_{j}$ be a non-empty set. Let $x \in \mathbb{R}^{n}$, and let $y^{o} \in C$, where $y^{o}$ has the minimal Euclidian distance to $x$, compare to all other points in $C$. For each, $\varepsilon>0$, Dykstra algorithm finds a point $y_{\varepsilon} \in C$, such that the Euclidian distance between $y^{o}$ and $y_{\varepsilon}$ is less than $\varepsilon$. We apply this algorithm in the following example 1.

\subsection{Examples}

\section{Example 1}

In this example we determine the premium for six classes. We compare some premium principles as described in section 3.2. Class $k$ contains $n_{k}$ insureds, where each has a probability $q_{k}$ for a claim. The mean and variance of a class $k$ claim are $\mu_{k}$ and $\sigma_{k}^{2}$, respectively. The mean and the variance of the total claims from class $k$ are $n_{k}=n_{k} q_{k}$ and $v_{k}^{2}=\mu_{k}^{2} q_{k}\left(1-q_{k}\right)+\sigma_{k}^{2} q_{k}$, respectively. The information of each class is shown in table 1 .

In Table 2 we present the premiums for each class according to the expectation principle, the variance principle, the uniform allocation and the semiuniform allocation.

Note that according to the semi-uniform allocation, the premium for class 3 is greater than the premium for class 4 , although the expected claim amount of class 3 is less than the expected claim amount in class 4 . Therefore, we use Theorem 2 to obtain the premiums that are based on the semi-uniform allocation, i.e., $r_{i}=1$ for each $i=1, \ldots, 6$. In column 6 we present the premium for each class assuming $a_{i}=0.1$ for each $i=1, \ldots, 6$ (Semi-Uniform BMS0). In the last column we assume that $a_{i}=0$, that is, a difference of at least $10 \%$ between the classes (Semi-Uniform BMS1). The premiums in BMS0 and BMS1 were obtained by the Dykstra algorithm.

The difference between premiums in the uniform allocation is the same as the difference between the corresponding the expected values of the claims. 
TABLE 1

DATA FOR EXAMPLE 1

\begin{tabular}{c|c|c|c|r|c|c}
\hline \hline Class & $\begin{array}{c}\text { Population } \\
\text { in class }\end{array}$ & $\begin{array}{c}\text { Claim } \\
\text { Probability }\end{array}$ & & & Mean & Variance \\
\hline$k$ & $n_{k}$ & $q_{k}$ & \multicolumn{1}{c|}{$\mu_{k}$} & $\sigma_{k}^{2}$ & $\eta_{k}=q_{k} \mu_{k}$ & $v_{k}^{2}=\mu_{k}^{2} q_{k}\left(1-q_{k}\right)+\sigma_{k}^{2} q_{k}$ \\
\hline 1 & 4000 & 0.050 & $2,100.00$ & 100,000 & 105 & 214,475 \\
2 & 2200 & 0.100 & $10,000.00$ & 200,000 & 1,000 & $9,020,000$ \\
3 & 800 & 0.210 & $13,000.00$ & 100,000 & 2,730 & $28,058,100$ \\
4 & 1500 & 0.185 & $15,000.00$ & $6,000,000$ & 2,775 & $35,034,375$ \\
5 & 800 & 0.250 & $17,000.00$ & $8,000,000$ & 4,250 & $56,187,500$ \\
6 & 500 & 0.300 & $19,000.00$ & $7,000,000$ & 5,700 & $77,910,000$ \\
\hline \hline
\end{tabular}

TABLE 2

OPTIMAL PREMIUMS ALLOCATION

\begin{tabular}{c|c|c|c|c|c|c}
\hline \hline Class & $\begin{array}{c}\text { Expected } \\
\text { Principle }\end{array}$ & $\begin{array}{c}\text { Variance } \\
\text { principle }\end{array}$ & $\begin{array}{c}\text { Uniform } \\
\text { allocation }\end{array}$ & $\begin{array}{c}\text { Semi-Uniform } \\
\text { allocation }\end{array}$ & $\begin{array}{c}\text { Semi-Uniform } \\
\text { BMS0 }\end{array}$ & $\begin{array}{c}\text { Semi-Uniform } \\
\text { BMS1 }\end{array}$ \\
\hline$k$ & & & & & $a_{i}=0$ & $a_{i}=0.1$ \\
\hline 1 & 109.81 & 105.83 & 176.13 & 134.05 & 134.25 & 138.05 \\
2 & $1,045.81$ & $1,035.01$ & $1,071.13$ & $1,052.81$ & $1,053.20$ & $1,060.10$ \\
3 & $2,855.06$ & $2,838.90$ & $2,801.13$ & $2,875.23$ & $2,858.20$ & $2,658.40$ \\
4 & $2,902.13$ & $2,910.98$ & $2,846.13$ & $2,852.45$ & $2,858.20$ & $2,924.20$ \\
5 & $4,444.70$ & $4,468.08$ & $4,321.13$ & $4,395.23$ & $4,395.50$ & $4,414.90$ \\
6 & $5,961.12$ & $6,002.39$ & $5,771.13$ & $5,932.36$ & $5,934.30$ & $5,964.40$ \\
\hline \hline
\end{tabular}

This is due to the fact that the uniform principle does not take into account the number of insureds in each class nor the variance. Therefore, the premium for class one is the biggest compare to all the other premium principles while the premium of the sixth class is the smallest among all other premium principles.

In the semi-uniform principle the variance is shared among all the insureds in the same class. Thus, the premiums for classes 1 and 2, which are the classes with the biggest number of insurdes, are reduced with respect to the uniform allocation, while the premiums for the classes with smaller number of insureds are higher compared to the uniform allocation. This phenomenon occurs also in the expected principle premium. The biggest differences among the premiums occur when considering the variance principle. In this case both the class size and the variance of the claim size are taken into account. Therefore, the biggest 
difference between the premiums for class 1 and class 6 is obtained in the variance principle. The smallest difference between the premiums for class 1 and class 6 is obtained in the uniform allocation. Note that the variance of a policyholder in class 6 is $\frac{77,910,000}{500}=155,820$. This value is over 2900 times of the variance of a policyholder in class 1 and over twice of the variance of a policyholder who is in class 5 .

\section{Example 2}

The goal of this example is to compare the methods presented in Theorem 1 to price bonus-malus systems, to known methods.

Lemaire (1995, Chapter 10) considers a fair system in the Bayesian sense. The number of claims for each policyholder during a year is assumed to be a Poisson distributed random variable with parameter $\Lambda$. $\Lambda$ is assumed to be a random variable, with Gamma distribution, with shape parameter $\alpha$ and scale parameter $\tau$. That is the density function of $\Lambda$ is: $f_{\Lambda}(\lambda)=\frac{e^{-\tau \lambda}(\tau \lambda)^{a-1} \tau}{\Gamma(a)}, \lambda>0$, and $E[\Lambda]=\frac{a}{\tau}$. Let $K_{i}$ be the number of claims of the policyholder in year $i$, $i=1,2, \cdots$, . Given the claim frequency in the first $t$ years, that is given that $K_{i}=k_{i}, i=1, \cdots, t$, the conditional density of $\Lambda$ is Gamma with shape parameter $k_{t}=a+\sum_{i=1}^{t} k_{i}$, scale parameter $\tau+t$ and expectation $\frac{a+\sum_{i=1}^{t} k_{i}}{\tau+t}$.

The premium in year $t+1$ is based on the number of claims during the first $t$ year and it is: $100 \frac{\tau\left(a+\sum_{i=1}^{t} k_{i}\right)}{a(t+\tau)}$. Thus, the premium for the first year is 100 .

Lemaire developed the above pricing system to a portfolio of 106,965 policyholders, where 96,978 policyholders having a history of no claims, 9240 with 1 claim, 704 with 2 claims and 43 policyholders with 3 claims. The data is taken from Lemaire (1995, Chapter 3). The average frequency of claims is 0.1011 and variance is 0.1074 . From these numbers we conclude that $a=1.6049$, $\tau=15.8778$.

The premiums obtained by Lemaire (1995) for 7 years for policyholders having $k$ claims in $t$ years are exhibited in Table 3.

To derive the premiums by the methods discussed in section 3.2, we consider eight portfolios, numbered $0, \cdots, 7$, that is, for each year in Lemaire's example we consider a different portfolio. Each portfolio consists of four classes indexed from 0 to 3 . All the eight portfolios contain the same number of policyholders for each class. The numbers of policyholders from class $j$ is $n_{j}, j=0,1,2,3$, where $n_{0}=96,978, n_{1}=9,240, n_{2}=704, n_{3}=43$.

Portfolio 0 is homogeneous, that is the expectation and the variance of the claim amount of all policyholders are $\frac{a}{\tau}$ and $\frac{a}{\tau^{2}}$ respectively, and the number of policyholders is $n=106,965$. Thus the premium for a given risk level $\alpha$ is $\pi_{o}=\frac{a}{\tau}+z_{1-\alpha} \frac{\sigma}{\sqrt{n}}$. 
TABLE 3

BonUS MALUS SYSTEM ACCORDING TO LEMAIRE (1995)

\begin{tabular}{|c|c|c|c|c|}
\hline year & 0 & 1 & 2 & 3 \\
\hline 0 & 100.00 & & & \\
\hline 1 & 94.08 & 152.69 & 211.31 & 269.93 \\
\hline 2 & 88.81 & 144.15 & 199.49 & 254.83 \\
\hline 3 & 84.11 & 136.52 & 188.92 & 241.33 \\
\hline 4 & 79.88 & 129.65 & 179.42 & 229.19 \\
\hline 5 & 76.05 & 123.44 & 170.82 & 218.21 \\
\hline 6 & 72.57 & 117.80 & 163.02 & 208.24 \\
\hline 7 & 69.40 & 112.65 & 155.89 & 199.14 \\
\hline
\end{tabular}

TABLE 4

BMS ACCORDING TO THE UNIFORM ALLOCATION

\begin{tabular}{|c|rrrr|}
\hline \multicolumn{1}{|r|}{ claims } & \multicolumn{1}{c|}{$\mathbf{0}$} & $\mathbf{1}$ & $\mathbf{2}$ & $\mathbf{3}$ \\
\hline 0 & 100.00 & & & \\
1 & 94.09 & 152.38 & 210.67 & 268.96 \\
2 & 88.83 & 143.86 & 198.89 & 253.92 \\
3 & 84.12 & 136.24 & 188.35 & 240.47 \\
4 & 79.89 & 129.38 & 178.88 & 228.37 \\
5 & 76.06 & 123.19 & 170.31 & 217.43 \\
6 & 72.59 & 117.56 & 162.52 & 207.49 \\
7 & 69.41 & 112.42 & 155.42 & 198.42 \\
\hline \hline
\end{tabular}

TABLE 5

BMS ACCORDING to THE SEMI-UNIFORM ALLOCATION

\begin{tabular}{|c|c|c|c|c|}
\hline year claims & 0 & 1 & 2 & 3 \\
\hline 0 & 100.00 & & & \\
\hline 1 & 93.14 & 153.58 & 224.87 & 495.18 \\
\hline 2 & 87.93 & 144.99 & 212.29 & 467.48 \\
\hline 3 & 83.27 & 137.31 & 201.04 & 442.72 \\
\hline 4 & 79.08 & 130.41 & 190.93 & 420.44 \\
\hline 5 & 75.29 & 124.16 & 181.78 & 400.30 \\
\hline 6 & 71.85 & 118.48 & 173.48 & 382.01 \\
\hline 7 & 68.71 & 113.30 & 165.89 & 365.31 \\
\hline
\end{tabular}


In the $j$ th portfolio the mean and the variance of the claim amount of class $k$ are equal to $\frac{a+k}{\tau+j}$ and $\frac{a+k}{(\tau+j)^{2}}$ respectively, $j=1, \cdots, 7, k=0,1,2,3$.

The risk level is chosen to be $1 \%$.

For $j=1, \cdots, 7, k=0,1,2,3$, let $\pi_{j, k}$ be the premium for class $k$ in the $j$ th portfolio. Table 4 exhibits the values of $100 \frac{\pi_{j, k}}{\pi_{0}}$ when $\pi_{j, k}$ is calculating according to the uniform allocation. Table 5 exhibits the values of $100 \frac{\pi_{j, k}}{\pi_{0}}$ when $\pi_{j, k}$ is calculating according to the semi-uniform allocation.

We see that tables 3 and 4 are quite similar. In table 5, the dangerous policyholders are punished much severely. As in example 1, the impact of the variance of a single policyholder is emphasized in the semi-uniform allocation and not in the uniform allocation.

Note that the premium levels for the different classes in the uniform allocation are similar to Lemaire's results. In the semi-uniform allocation the dangerous policyholders (class 3 ) are punished more severely than in Lemaire's example, while non-dangerous policyholders have a greater bonus than in Lamire's example. This is due to the fact that the number of policyholders in the nondangerous class 0 is higher than the number of policyholders in the dangerous class-class 3 , thus the share of each policyholder in class 0 in the total variance is much less than in class 3 .

Thus, choosing appropriate premium allocation, such as the semi-uniform principle or the variance principle, the more dangerous classes pay higher premium and the less dangerous policyholders pay less than in the Bayes pricing system. Note also that the methods applied in the paper, enable us to take into account also the claims' size, and not only the claims' frequency as in the Lemaire's example.

\section{THE DUAL PROBLEM}

In Section 3 we found the premiums for each class such that the expected squared deviation between the collected premiums and the payments of the insurer is minimized, subject to a given risk level. In this section we consider the dual problem: as stated in Theorem 3: The minimization of the risk level under the constraint that the expected squared deviation between the collected premiums and the insurer's payments is given.

Let, $\tilde{\pi}_{i}=n_{i} \pi_{i} \quad \tilde{\mu}_{i}=n_{i} \mu_{i}, \quad \tilde{\pi}=\left(\tilde{\pi}_{1}, \cdots, \tilde{\pi}_{k}\right)$.

\subsection{The dual optimal allocation}

Theorem 3
Let $r_{1}, \cdots, r_{k}$ and $\tilde{A}$ be positive numbers, where $\tilde{A} \geq \sum_{i=1}^{k} \frac{n_{i} \sigma_{i}^{2}}{r_{i}}$. 
The minimization problem

$$
\text { (VII) }\left\{\begin{array}{l}
\min _{\tilde{\pi}}\left\{P\left(S>\sum_{i=1}^{k} \tilde{\pi}_{i}\right)\right\} \\
\text { s.t. } \sum_{i=1}^{k}\left[\frac{1}{r_{i}} E\left(S_{i}-\tilde{\pi}_{i}\right)^{2}\right]=\tilde{A} \\
\tilde{\mu}_{i} \leq \tilde{\pi}_{i} \quad i=1, \ldots, k
\end{array}\right.
$$

has a unique solution which is

$$
\pi_{i}=\mu_{i}+r_{i} \sqrt{\frac{A}{r}} \quad \forall i=1, \ldots, k
$$

where $r=\sum_{j=1}^{k} r_{j}$ and $A=\tilde{A}-\sum_{i=1}^{k} \frac{n_{i} \sigma_{i}^{2}}{r_{i}}$.

\section{Proof}

$$
P\left(S>\sum_{i=1}^{k} \tilde{\pi}_{i}\right)=P\left(\frac{S-\mu}{\sigma}>\frac{\sum_{i=1}^{k} \tilde{\pi}_{i}-\sum_{i=1}^{k} \tilde{\mu}_{i}}{\sigma}\right) .
$$

Minimizing the left-hand side of (15) with respect to $\left(\tilde{\pi}_{i}, \cdots, \tilde{\pi}_{k}\right)$ is equivalent to finding the maximum of $\frac{\sum_{i=1}^{k}\left(\tilde{\pi}_{i}-\tilde{\mu}_{i}\right)}{\sigma}$ or, equivalently to the maximization of $\sum_{i=1}^{k}\left(\tilde{\pi}_{i}-\tilde{\mu}_{i}\right)$ (since $\sigma>0$ is a constant). The constraint can be rewritten as in Theorem 1. Thus, problem (VII) is equivalent to the following problem (VIII):

$$
\text { (VIII) }\left\{\begin{array}{l}
\max _{\tilde{\pi}}\left\{\sum_{i=1}^{k}\left(\tilde{\pi}_{i}-\tilde{\mu}_{i}\right)\right\} \\
\text { s.t. } \sum_{i=1}^{k} \frac{n_{i} \sigma_{i}^{2}}{r_{i}}+\sum_{i=1}^{k} \frac{1}{r_{i}}\left(\tilde{\pi}_{i}-\tilde{\mu}_{i}\right)^{2}=\tilde{A} . \\
\tilde{\mu}_{i} \leq \tilde{\pi}_{i} \quad i=1, \ldots, k
\end{array}\right.
$$

Let $X_{i}=\tilde{\pi}_{i}-\tilde{\mu}_{i}$, and $A=\tilde{A}-\sum_{i=1}^{k} \frac{n_{i} \sigma_{i}^{2}}{r_{i}}$. Problem (VIII) can then be rewritten as
follows 


$$
\text { (IX) }\left\{\begin{array}{l}
\max _{\tilde{\pi}}\left\{\sum_{i=1}^{k} X_{i}\right\} \\
\text { s.t. } \sum_{i=1}^{k} \frac{1}{r_{i}} X_{i}^{2}-A=0 \\
X_{i} \geq 0 \quad i=1, \ldots, k .
\end{array}\right.
$$

To solve this problem we use Lagrange multipliers. Let

$$
G(X, \lambda)=\sum_{i=1}^{k} X_{i}+\lambda\left(\sum_{i=1}^{k} \frac{1}{r_{i}} X_{i}^{2}-A\right) .
$$

Differentiate $G(X)$ with respect to $\left(X_{1}, \cdots, X_{k}\right)$ and $\lambda$ yields a system of $k+1$ equations:

$$
\left\{\begin{array}{l}
\frac{\partial G(X, \lambda)}{\partial X_{i}}=1+2 \lambda \frac{X_{i}}{r_{i}}=0 \quad \forall i=1, \ldots, k \\
\frac{\partial G(X, \lambda)}{\partial \lambda}=\sum_{i=1}^{k} \frac{X_{i}^{2}}{r_{i}}-A=0
\end{array}\right.
$$

From the first $k$ equations of (16) we obtain:

$$
X_{i}=-\frac{r_{i}}{2 \lambda} \quad \forall i=1, \ldots, k \text {. }
$$

Since $X_{i} \geq 0$ then $\lambda$ has to be negative. Substituting (17) in the last equation of (16) yields,

$$
\begin{aligned}
& \sum_{i=1}^{k} \frac{\left(-\frac{r_{i}}{2 \lambda}\right)^{2}}{r_{i}}=A \\
& \Rightarrow \frac{1}{4 A} \sum_{i=1}^{k} r_{i}=\lambda^{2}
\end{aligned}
$$

Since $\lambda<0$, substituting the negative root of $\lambda$ from (18) in the first $k$ equations of (16) yields a unique solution which is

$$
X_{i}^{*}=-\frac{r_{i}}{2 \lambda}=r_{i} \sqrt{\frac{A}{r}} \quad \forall i=1, \ldots, k .
$$

Next we confirm that the point $X^{*}=\left(X_{1}^{*}, \ldots, X_{k}^{*}\right)$ is a maximum. Let $\varepsilon_{1}, \ldots, \varepsilon_{k} \in \mathbb{R}$ be $k$ arbitrary scalars. The point $X^{*}$ is the solution of Problem (IX), if, and only if 


$$
\sum_{i=1}^{k} y_{i}<\sum_{i=1}^{k} X_{i}^{*}
$$

for any point $y=\left(y_{1}, \ldots, y_{k}\right) \in \mathbb{R}^{k}$ such that $y_{i} \geq 0, i=1, \cdots, k$ and such that :

$$
\sum_{i=1}^{k} \frac{1}{r_{i}} y_{i}^{2}-A=0 \text {. }
$$

Let $y_{i}=X_{1}^{*}+r_{i} \varepsilon_{i} \quad \forall i=1, \ldots, k$. Hence, (20) implies

$$
\sum_{i=1}^{k} \frac{1}{r_{i}}\left(X_{i}^{*}+r_{i} \varepsilon_{i}\right)^{2}=A .
$$

Substituting the values of $X_{1}^{*}$ as given in (19) yields:

$$
\begin{aligned}
& \sum_{i=1}^{k} \frac{1}{r_{i}}\left(r_{i} \sqrt{\frac{A}{r}}+r_{i} \varepsilon_{i}\right)^{2}=A \\
& \Rightarrow \sum_{i=1}^{k} r_{i}\left(\frac{A}{r}+2 \sqrt{\frac{A}{r}} \varepsilon_{i}+\varepsilon_{i}^{2}\right)=A \\
& \Rightarrow 2 \sqrt{\frac{A}{r}} \sum_{i=1}^{k} r_{i} \varepsilon_{i}+\sum_{i=1}^{k} r_{i} \varepsilon_{i}^{2}=0 \\
& \Rightarrow \sum_{i=1}^{k} r_{i} \varepsilon_{i}=-\frac{1}{2} \sqrt{\frac{r}{A}} \sum_{i=1}^{k} r_{i} \varepsilon_{i}^{2}<0
\end{aligned}
$$

Therefore,

$$
\sum_{i=1}^{k} y_{i}=\sum_{i=1}^{k}\left(X_{i}^{*}+r_{i} \varepsilon_{i}\right)=\sum_{i=1}^{k} X_{i}^{*}+\sum_{i=1}^{k} r_{i} \varepsilon_{i}<\sum_{i=1}^{k} X_{i}^{*} .
$$

Thus the optimal premium for class $i$ is :

$$
\pi_{i}=\mu_{i}+r_{i} \sqrt{\frac{A}{r}} \quad \forall i=1, \ldots, k .
$$

\section{Corollary 2} By changing the first constraint of problem (VII) into $\sum_{i=1}^{k}\left[\frac{1}{r_{i}} E\left(S_{i}-\tilde{\pi}_{i}\right)^{2}\right] \leq \tilde{A}$ the
unique solution does not change.

The proof is similar to the proof of Corollary 1 . 


\subsection{The dual problem with ordered premium}

In this subsection we determine the premium for each class such that the premiums are minimized the risk level under the constraint that the expected squared deviation between the collected premiums and the insurer's payments is given. In addition, we consider the case where the insurer or the regulator, wants to grade the premiums for each class such that the premium will increase as its risk increases.

\section{Theorem 4}

Let $a_{1}, \ldots, a_{k-1}, r_{1}, \ldots, r_{k}$ be positive scalars. Such that

$$
\left(1+a_{i}\right) \mu_{i} \leq \mu_{i+1} \quad i=1, \ldots, k-1
$$

The maximization problem

$$
\text { (X) }\left\{\begin{array}{l}
\max _{\tilde{\pi}}\left\{\sum_{i=1}^{k}\left(n_{i} \pi_{i}-n_{i} \mu_{i}\right)\right\} \\
\text { s.t. } \sum_{i=1}^{k} \frac{n_{i} \sigma_{i}^{2}}{r_{i}}+\sum_{i=1}^{k} \frac{1}{r_{i}}\left(n_{i} \pi_{i}-n_{i} \mu_{i}\right)^{2}=\tilde{A} \\
\left(1+a_{i}\right) \pi_{i} \leq \pi_{i+1} \quad i=1, \ldots, k-1 \\
\mu_{i} \leq \pi_{i} \quad i=1, \ldots, k
\end{array}\right.
$$

has at least one solution.

\section{Proof}

By substituting

$$
X_{i}=n_{i} \pi_{i}-n_{i} \mu_{i}
$$

the problem $(\mathrm{X})$ becomes

$$
\text { (XI) }\left\{\begin{array}{l}
\max _{\tilde{\pi}}\left\{\sum_{i=1}^{k} X_{i}\right\} \\
\text { s.t. (a) } \sum_{i=1}^{k} \frac{1}{r_{i}} X_{i}^{2}=A \\
\text { (b) }\left(1+a_{i}\right)\left(\frac{X_{i}}{n_{i}}+\mu_{i}\right) \leq \frac{X_{i+1}}{n_{i+1}}+\mu_{i+1} \quad i=1, \ldots, k-1 \\
\text { (c) } 0 \leq X_{i} \quad i=1, \ldots, k
\end{array}\right.
$$

The vector $X=\left(X_{1}, \ldots, X_{k-1}, X_{k}\right)=\left(0, \ldots, 0, \sqrt{A r_{k}}\right)$ is a feasible solution for the constraints of problem (XI). Denote by $C$ the intersection of (a), (b) and (c) 
in problem (XI). Hence, the set $C$ is a non-empty and close set. Since the objective function $f(X)=\sum_{i=1}^{k} X_{i}$ is a continuous function, it follows that $f$ has a minimum and a maximum in $C$.

Remark: We did not succeed to prove that there is a unique point where the maximum in problem $(X)$ is achieved, that is there might be several points where the global maximum is achieved. However, in the examples we tested, we obtained a unique solution. Since the constraints are not-linear, we find the solution by using the well known Lagrange's multipliers method, see (Apostol, (1969), chapter 9).

\section{Example 3:}

The example refers to theorems 3 and 4 . We consider three classes. Class $k$ contains $n_{k}$ policyholders, where each has a probability $q_{k}$ for a claim. The mean and variance of a class $k$ claim are $\mu_{k}$ and $\sigma_{k}^{2}$, respectively. The mean and the variance of the total claims from class $k$ are, $\eta_{k}$ and $v_{k}^{2}$ respectively, where $\eta_{k}=q_{k} \mu_{k}$ and $v_{k}^{2}=\mu_{k}^{2} q_{k}\left(1-q_{k}\right)+\sigma_{k}^{2} q_{k}$. The weight for class $i$ is $r_{i}=1 / n_{i}$. The information of each class is shown in table 6 . We assume that $A=10,000,000$. In table 7, we present the premiums for each class according to the data in table 6.

Assume the insurer wants a BMS where the premiums are at least doubled between the classes. The premiums that are obtained by theorem 3 do not satisfy

TABLE 6

DATA FOR EXAMPLE 3.

\begin{tabular}{c|c|c|c|c|r|r|r}
\hline \hline Class & Weight & $\begin{array}{c}\text { Population } \\
\text { in class }\end{array}$ & $\begin{array}{c}\text { Claim } \\
\text { Probability }\end{array}$ & & & Mean & Variance \\
\hline$k$ & $r_{i}$ & $n_{k}$ & $q_{k}$ & $\mu_{k}$ & $\sigma_{k}^{2}$ & $\eta_{k}$ & \multicolumn{1}{c}{$v_{k}^{2}$} \\
\hline 1 & $1 / 6000$ & 6000 & 0.050 & $2,100.00$ & 500,000 & 105.00 & 234,475 \\
2 & $1 / 1500$ & 1500 & 0.108 & $11,000.00$ & $3,000,000$ & $1,188.00$ & $11,980,656$ \\
3 & $1 / 1000$ & 1000 & 0.145 & $16,500.00$ & $1,000,000$ & $2,392.50$ & $33,897,193$ \\
\hline \hline
\end{tabular}

TABLE 7

OPTIMAL PREMIUMS FOR THE DUAL PROBLEM

\begin{tabular}{c|c|c}
\hline \hline Class $\boldsymbol{k}$ & $\begin{array}{c}\text { Premiums by } \\
\text { theorem 3 }\end{array}$ & $\begin{array}{c}\text { BMS with doubled } \\
\text { premiums }\end{array}$ \\
\hline 1 & 139.30 & 133.76 \\
2 & $1,222.30$ & $1,212.04$ \\
3 & $2,426.80$ & $2,457.09$ \\
\hline \hline
\end{tabular}


the demand for $100 \%$ difference between the classes, since the difference between classes 2 and 3 is about $98.5 \%$. For this reason, we calculate the premiums for the appropriate BMS.

\section{ACKNOWLEDGEMENTS}

The authors are grateful to the anonymous referee for his/her comments that greatly improved the original manuscript.

Yaniv Zaks acknowledges the financial support of the Zimerman foundation for the study of banking and finance.

\section{REFERENCES}

Baptiste J., Urruty, H. and Lemarechal, C. (1993) Convex analysis and minimization algorithms I. Springer-Verlag, Berlin, Germany.

Bowers, N.L., Gerber, H.U., Hickman, J.C., Jones, D.A. and Nesbitt, C.J. (1997) Actuarial Mathematics. The Society of Actuaries, U.S.A.

Censor, Y. and ReICH, S. (1998) The Dykstra algorithm with Bregman projections, Communications in Applied Analysis, 2, 407-419.

DykstRA R.L. (1983) An Algorithm for restricted least squares regression, Journal of American Statistical Association, 78, 837-842.

Frangos, N.E. and VRontos, S.D. (2001) Design of optimal Bonus-Malus Systems with a frequency and a severity component on an individual basis in automobile insurance. Astin Bulletin, 31(1), 5-26.

Goovaerts, M.J., De Vylder, F. and HaEzendonck, J. (1984) Insurance premiums. Elsevier Science Publishers B.V., Amsterdam, The Netherlands.

Kliger, D. and Levikson, B. (1998) Pricing Insurance contracts - An economic viewpoint. Insurance Mathematics and Economics, 22(3), 243-249.

Lemaire J. (1995) Bonus-Malus Systems in Automobile insurance. Kluwer Academic Publishers, Massachusetts, USA.

YANiv ZaKs, Esther Frostig And Benny LeVIKSON

Department of Statistics

University of Haifa

Israel 


\section{APPENDIX}

\section{Proof of lemma 1:}

Let $x \in \mathbb{R}^{m}(x \neq 0)$ and let $z=P x$. Since $P$ is nonsingular and $x \neq 0$ then $z \neq 0$. Since $A$ is a positive definite matrix, $x^{t} A x>0$ for each $x \neq 0$ and especially $z^{t} A z>0$. Therefore

$$
\begin{gathered}
x^{t} B x=x^{t} P^{t} A P x= \\
=(P x)^{t} A(P x)=z^{t} A z>0
\end{gathered}
$$

\section{Proof of Lemma 2:}

A matrix is a positive definite matrix if and only if each one of its leading principal minors is positive. The lemma is proved by induction on $m$.

Clearly the Lemma holds for $m=1$. Suppose that $A_{m-1}$ is a positive definite matrix. Thus, all its main minors are positive. Hence it is enough to show $\left|A_{m}\right|>0$, where $|A|$ is the determinant of $A$. Note that $\left|A_{m}\right|=\left|D_{m}\right|+\left|E_{m}\right|$, where

$$
\left.D_{m}=\left|\left[\begin{array}{cccc}
1+a_{1} & & & 0 \\
& \ddots & 1 & \vdots \\
& & 1+a_{m-1} & 0 \\
1 & & & a_{m}
\end{array}\right]\right| \text {, and } E_{m}=\mid \begin{array}{cccc}
1+a_{1} & & & 1 \\
& \ddots & 1 & \vdots \\
& & 1+a_{m-1} & \vdots \\
1 & & & 1
\end{array}\right] \mid \text {. }
$$

Evaluating the determinant of $D_{m}$ according to the last column yields:

$$
\left|D_{n}\right|=(-1)^{2 m} a_{m}\left|A_{m-1}\right|>0,
$$

where the inequality holds since $a_{m}>0$ and since by the induction hypothesis $\left|A_{m-1}\right|>0$.

To evaluate $\left|E_{m}\right|$ we subtract the last column from each of the other columns, and we obtain

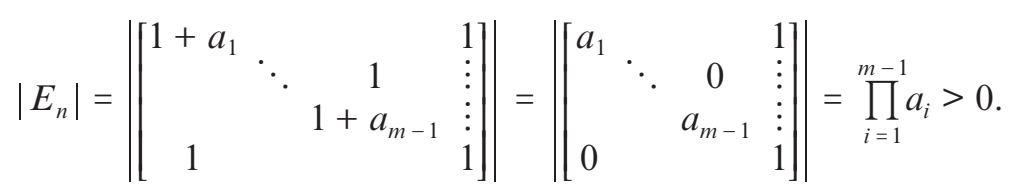

Thus, $\left|A_{m}\right|>0$. 